\title{
Cheap-Talk and Cooperation in a Society*
}

\author{
AKIHIKo MatSUI \\ Department of Economics, University of Pennsyluania, \\ Philadelphia, Pennsylvania 19104-6297
}

Received October 10, 1989; revised November 21, 1990

\begin{abstract}
This paper considers a large population randomly matched to play a game of common interest with cheap-talk. We apply a noncooperative solution concept to this society. A strategy distribution $g$ is accessible from another distribution $f$ if there is a path from $f$ to $g$ the direction of which is always a best response to the present distribution. A cyclically stable set is a set of strategy distributions which is closed under accessibility and of which any two members are mutually accessible. It is shown that a unique cyclically stable set exists and contains only Pareto optimal outcomes. Iournal of Fronomic Literature Classification Numbers: 022, 026. 1991 Academic Press, Inc
\end{abstract}

\section{INTRODUCTION}

Does cheap-talk matter? Does costless communication necessarily yield Pareto optimal outcomes? Can we have a foundation of cooperative behavior in the context of noncooperative game theory? The answer to these questions depend, of course, on the specific way of modeling the interaction and, most importantly, on the solution concept applied. This paper uses a new solution concept adopting a societal-dynamics perspective, which yield quite different results than more "traditional" ones. Let us first briefly review some of the existing concepts.

In the field of noncooperative game theory, every solution concept requires that players make rational choices based on their beliefs, and that those beliefs satisfy a certain consistency. The criteria of rational choiccs and consistency of beliefs vary from one concept to another. For example, in Nash equilibrium (Nash [11]), each player maximizes his/her own (unconditional) expected payoff given the other players' strategies. Sequential equilibrium (Kreps and Wilson [7]) requires, in addition to those conditions required in Nash equilibrium, that each player chooses an action

* The author thanks Professor ltzhak Gilboa for his guidance. Thanks are also given to seminar participants at Hitotsubashi University, Northwestern University, University of Pennsylvania, and Stanford University, as well as Professor Mamoru Kaneko, and a referee and an associate editor of this journal for helpful comments and references. 
that maximizes his/her conditional expected payoff at every information set, and that the conditional belief system is consistent with the equilibrium strategy profile.

Regarding normal form games, every equilibrium concept requires that players' beliefs about the other players' strategies must coincide with the "actual" strategies they plan to take. On the other hand, rationalzability (Bcrnhcim [3], Pcarce [12]) allows two players' belicfs to differ from the actual strategies as long as they are derived in a rational manner.

In spite of such a wide spectrum, there is a common assumption underlying these concepts, namely, that players' systems of beliefs and strategies do not change throughout the entire game. The game is played exactly once (if it is a repeated game, the repetition occurs once), and if they change their beliefs or strategies, the changes are incorporated in larger systems of beliefs and strategies. In this sense, each player is treated as if he/she had a complete contingent plan of beliefs and strategies. The stability of strategies discussed in this context is called strategic stability.

The analysis of cheap-talk models in this context raises some difficulties. Consider the game in Fig. 1. Though cooperation, namely the play $(L, L)$. has been prescribed as a unique outcome under unlimited communication (see, for instance, Bernheim, Peleg, and Whinston [4]), to the best of my knowledge, no purely noncooperative solution concept discussed in the literature has successfully excluded $(R, R)$ as an outcome. Indeed, even with cheap-talk, it is doubtful that $(L, L)$ is always chosen when $(R, R)$ is oriented. The reason is the following. If, say, player 1 wants to make a joint deviation to $(L, L)$, he must persuade player 2 that he plans to take $L$ with the probability of at least a half. Even if he is not sure that she is persuaded, he always gains by the persuasion if he sticks to $R$. Knowing this, she might conclude that it is safe to keep playing $R$. Hence, cheap-talk is not credible. On the other hand, if it is common knowledge that player 2 thinks the persuasion credible, then player 1 is willing to persuade her and take $L$. Both of these scenarios make some sense. Therefore, it seems that one must arbitrarily assume a certain degree of credibility of cheap-talk in

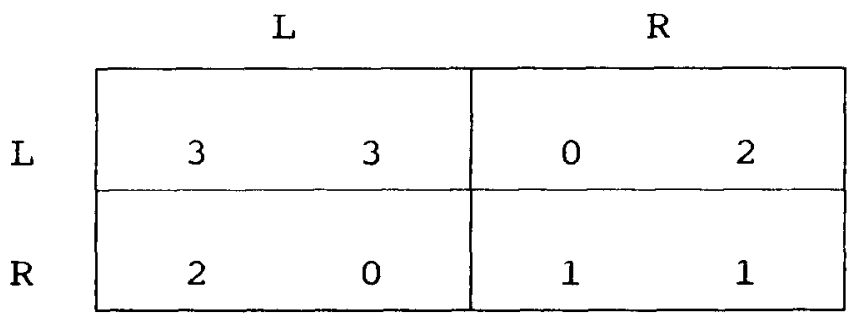


refining equilibrium. We cannot escape from this type of arbitrariness if we try to provide cheap-talk with some meaning in the context of strategic stability. We will see that if one adopts a societal-dynamics perspective, a high degree of credibility may be attained, rather than assumed, in the course of a movement of a behavior pattern.'

In many daily life situations, people do not know and/or do not care about the entire structure of the game. Nevertheless, they behave so as to maximize their payoffs. In order to behave optimally, all one has to know are one's own payoff and the opponents' strategies, or, to be precise, the expected payoff from each action available. One of various plausible scenarios explaining how they learn to behave optimally is that the game is repeated many times, and people determine their actions by trial and error. ${ }^{2}$ In this process, since the behavior pattern typically changes as time goes on, the belief system changes as well. Social stability refers to the stability of a stationary point in this repeated situation. Gilboa and Matsui [6] suggest a new solution concept called cyclically stable set on the basis of this way of viewing the world. This solution concept is applied to societies with infinitely may individuals who are matched randomly to play a single game. In the course of a long time repetition, the behavior pattern of society changes gradually in the direction of a best response. Its trajectory is referred to as a directly accessible path. A strategy distribution is accessible from another if either (i) there is a directly accessible path from the latter to any neighborhood of the former, or (ii) the former is accessible from another which is accessible from the latter. ${ }^{3}$ A cyclically stable set is

\footnotetext{
${ }^{1}$ Farrell [5] obtained a result in which cheap-talk matters in refining equilibrium in the class of signalling games. He considered situations in which players share sufficiently rich language, and players are better off by being honest if payoffs are the same. A message or a "neologism" is credible if one using the neologism does not have an incentive to tell a lie, assuming that the receiver of messages believes it. The neologism-proof equilibrium is an equilibrium in which a sender of messages does not have an incentive to use "credible neologism" to separate himself from other types. If, however, one generalizes his concept of neologismproof equilibrium to situations in which cheap-talk is used to separate actions instead of types, then it is subject to the same problem of arbitrariness. In addition to this, honesty, which is assumed to be preferred to dishonesty in his analysis, is also attained in ours. Honesty is not justified a priori even if the players share a rich enough language since it is often the case with our daily conversation that an unbelieved false message, which is considered as a mere joke, may not cause disutility. Rabin [13] shares the same problem with Farrell.

${ }^{2}$ In order to find a best response in a multi-stage game, one has to know what would be taken in unreached information sets. A possible scenario concerning how players acquire the information is that a negligible portion of individuals make experiments at each instance, and the obtained information spreads in the population.

"There are several versions of accessibility in Gilboa and Matsui [6], though all of them incorporate the idea that the behavior pattern moves in the direction of a best response to the current strategy distribution.
} 
a set of strategy distributions which is closed under accessibility, and any two members of which are accessible from each other. An interpretation is that once the actual behavior pattern falls in the set, it never leaves the set, and any strategy distribution in the set may always be realized. In the present paper, we apply this concept to the class of games with cheap-talk.

In the main part of the paper, we confine our attention to two-by-two games of common intercst. Wc say that a game is of common interest if there exists a unique weakly (a fortiori a unique strongly) Pareto optimal outcome. Then we consider the following two-person two-stage game. In the first stage, players simultaneously announce actions. In the second stage, they choose their actions after observing the first stage announcements. Payoffs are determined only by the actions they take in the second stage. This two-stage game is called a "game with cheap-talk" since the actions taken in the first stage directly affect neither the payoffs nor the set of actions available to players in the second stage of the game. The second stage of the original game will be referred to as the game without cheaptalk.

In the context of social stability, we assume that this two-stage game itself is repeated in a large society with random matching. As time goes on, a norm endowing cheap-talk with a certain meaning may develop. In particular, such a norm may lead to a Pareto optimal outcome. Indeed, in the example of Fig. 1, cooperation is bound to emerge. While this fact is formally proven in the subsequent sections, its logic may be informally explained as follows.

Suppose that the initial behavior pattern is such that all the players announce $R$ in the first stage and take $R$ in the second stage no matter what is announced in the first stage. Note that this strategy is a best response to itself. Then, some people start trying a new strategy which prescribes $L$ in the first stage, $L$ in the second stage if both have announced $L$, and $R$ otherwise. By adopting this strategy, one gains three if he/she meets a person with the same strategy and gains one if he/she meets a person taking the original strategy. This strategy fares better than the original strategy as well as the "cheating" strategy, according to which one announces $L$ and takes $R$ in the second stage no matter what has been announced. ${ }^{4}$ Once it prevails, taking $R$ in the second stage can never be prescribed by a best response strategy (except in unreached information sets).

This verbal description essentialy corresponds to a formal argument in the subsequent sections. Its logic involves two important points concerning

\footnotetext{
${ }^{4}$ If, for example, the cheating strategy is in the initial behavior pattern, the strategy in question does not increase its frequency because it is exploited by the cheating strategy. According to our solution concept, however, the cheating strategy will be driven away at some point in time by the strategy honestly announcing and taking $R$.
} 
social stability as distinguished from strategic stability. First, we deal with situations in which people continue learning their opponents' strategies on the basis of what happens in the past as well as in the current world. Through this learning process, the role of cheap-talk gradualy evolves.

Second, a society we are interested in consists of many individuals, who are matched randomly to play the game with cheap-talk and are never matched again in the future so that they are not involved in strategic interaction such as punishment beyond a single matching. If the game is repeated many times between the same individuals, then it is unavoidable for the analysis to take into account the stratcgic interaction betwecn different two-stage games, and consequently the game should be considered as a (possibly infinitely) repeated one. In that case, some studies have shown that the optimal outcome is necessarily attained under some qualifications; among those studies are Aumann and Sorin [2] and Matsui [8]. The point of the present approach is that these long-term strategic considerations are not needed to explain cooperation.

The approach presented here is also different from evolutionarily stable strategy proposed by Maynard Smith and Price [10]. The main distinction is that their solution concept is point-valued, while ours is set-valued. A set-valued solution concept is more natural than a point-valued one in the context of social or evolutionary stability since it captures not only the stability of a point, but also the stability of a cyclical movement, if any. It turns out that our solution always exists, and that we always find a cyclically stable set which is accessible from any initial distribution (see the proof in [6]).

The rest of this paper is organized as follows. Section 2 presents some notations and definitions. Section 3 defines and discusses the notion of social stability. Section 4 gives the main theorem which basically states that in the two-by-two games of comon interest with cheap-talk only Pareto efficient outcomes are in cyclically stable sets. Section 5 gives some additional examples. Section 6 concludes the paper.

\section{Games of Common Interest with Cheap-Talk}

In a society there are two types of individuals, 1 and 2; each type consists of infinitely many anonymous individuals. They are matched randomly to take some actions. In each matching situation, one individual from each type is selected, and they play a two-stage game.

In the first stage of each game, type 1 and type 2 players simultaneously announce either $L$ or $R$. In the second stage, knowing what has been announced, they again simultaneously take either $L$ or $R$. Therefore, each individual has five information sets, $u_{i}^{0}, u_{i}^{1}, u_{i}^{2}, u_{i}^{3}$, and $u_{i}^{4}$ where $u_{i}^{0}$ is the 
information set in the first stage, and $u_{i}^{1}, u_{i}^{2}, u_{i}^{3}$, and $u_{i}^{4}$ are the information sets which are reached when the first stage announcements were $(L, L)$, $(L, R),(R, L)$, and $(R, R)$, respectively (the first and the second coordinates of each pair correspond to the announcements of type 1 and type 2 players, respectively). A pure strategy of type $i$ player $(i=1,2)$, $s_{i}=\left(s_{i}^{0}, s_{i}^{1}, s_{i}^{2}, s_{i}^{3}, s_{i}^{4}\right)$, is an element of $S_{i}=\{L, R\}^{5}$ where $s_{i}^{k}(k=0,1, \ldots, 4)$ is the action taken at the information set $u_{i}^{k}$. We write $S=S_{1} \times S_{2}$. We assume that a strategy distribution of type $i(i=1,2)$ is a behavior strategy, which is expressed as a quintuple $f_{i}=\left(f_{i}^{0}, f_{i}^{1}, f_{i}^{2}, f_{i}^{3}, f_{i}^{4}\right)$ in $[0,1]^{5}$ where $f_{i}^{k}(k=0, \ldots, 4)$ is a "local" strategy at $u_{i}^{k}$ which prescribes $L$ with probability $f_{i}^{k}$. Let $F_{i}$ be the set of all strategy distributions of type $i$. We write $F=F_{1} \times F_{2}$. We assume that $F$ is a subset of a 10-dimensional space endowed with the usual linear operations and the norm $\|\cdot\|$ defined by $\|f\|=\sup _{i, k}\left|f_{i}^{k}\right|$. Given $f$ in $F$ and a subset $G$ of $F$, we let $\left\|f^{\prime}-G\right\|=\inf _{g \in G}\|f-g\|$.

Given $f$ in $F$, there are two possible scenarios concerning choices of strategies by individuals. One is that $f_{i}^{0}$-portion of the entire population of type $i(i=1,2)$ takes the pure local strategy $L$ at $u_{i}^{0}$, and $f_{i}^{k}$-portion takes $L$ conditional on $u_{i}^{k}$ being reached. The other is that every type $i$ individual takes the same behavior strategy $f_{i}$. This distinction might not affect our analysis in the sequel. However, we find the former more appealing than the latter and prefer to keep it in mind. In considering the dynamic adjustment process, the current strategy distribution will be often referred to as the behavior pattern.

Given a pair of local strategies $\left(f_{1}^{k}, f_{2}^{k}\right)$ at information sets $\left(u_{1}^{k}, u_{2}^{k}\right)$ for $k \neq 0$, the expected payoff for type $i$ player given $u_{i}^{k}$ being reached $(i=1,2)$ is calculated as

$$
\pi_{i}\left(f_{i}^{k}, f_{j}^{k}\right)=f_{i}^{k} f_{j}^{k} a_{i}+f_{i}^{k}\left(1-f_{j}^{k}\right) b_{i}+\left(1-f_{i}^{k}\right) f_{j}^{k} c_{i}+\left(1-f_{i}^{k}\right)\left(1-f_{i}^{k}\right) d_{i},
$$

where $j$ denotes not $i(j=3-i)$ hereafter. The second stage of the game is given in Fig. 2. A game of common interest if there is a unique weakly

L $\quad$ R

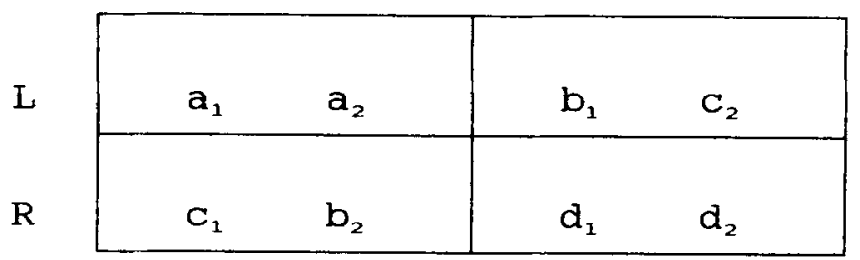


Pareto optimal outcome. In the present analysis, we assume without loss of generality that $a_{i}$ is strictly greater than $b_{i}, c_{i}$, and $d_{i}$ for $i=1,2$. Let

$$
q_{i}^{*}=\left(d_{i}-b_{i}\right) /\left(a_{i}-c_{i}+d_{i}-b_{i}\right), \quad i=1,2,
$$

if the denominator is positive, and $q_{i}^{*}=-\delta$ with $\delta>0$ otherwise. Then at $u_{i}^{k}(k \neq 0, i=1,2)$, we have

$$
\operatorname{argmax}_{f_{i}^{\prime} \in F_{i}} \pi_{i}\left(f_{i}^{\prime}, f_{j}^{k}\right) \begin{cases}=\{1\} & \text { if } f_{j}^{k}>q_{i}^{*}, \\ \in[0,1] & \text { if } f_{i}^{k}=q_{i}^{*}, \\ =\{0\} & \text { if } f_{j}^{k}<q_{i}^{*} .\end{cases}
$$

Note that if $q_{i}^{*}$ is nonpositive, it is always a best response for player $i$ to take $L$ in the second stage.

Given a strategy profile $f \in F$, the expected payoff for an individual of type $i(i=1,2)$ if he/she takes a behavior strategy $g_{i} \in F_{i}$ is

$$
\begin{aligned}
\Pi_{i}\left(f ; g_{i}\right)= & g_{i}^{0} f_{j}^{0} \pi_{i}\left(g_{i}^{1}, f_{j}^{1}\right)+g_{i}^{0}\left(1-f_{j}^{0}\right) \pi_{i}\left(g_{i}^{1+i}, f_{j}^{1+i}\right) \\
& +\left(1-g_{i}^{0}\right) f_{j}^{0} \pi_{i}\left(g_{i}^{4-i}, f_{i}^{4-i}\right) \\
& +\left(1-g_{i}^{0}\right)\left(1-f_{j}^{0}\right) \pi_{i}\left(g_{i}^{4}, f_{j}^{4}\right) .
\end{aligned}
$$

We may write $\Pi_{i}(f)=\Pi_{i}\left(f ; f_{i}\right)$ and $\Pi(f)=\left(\Pi_{1}(f), \Pi_{2}(f)\right)$.

Let $\operatorname{Br}_{i}(f)$ be the set of strategies for individuals of type $i(i=1,2)$ that are best responses to $f$; i.e.,

$$
\mathrm{Br}_{i}(f)=\operatorname{argmax}_{g_{i} \in F_{i}} \Pi_{i}\left(f ; g_{i}\right) .
$$

We may write $\operatorname{Br}(f)=\mathrm{Br}_{1}(f) \times \operatorname{Br}_{2}(f)$. Given $f$ in $F$, let $U_{c}(f)$ be the $\varepsilon$-neighborhood of $f$; i.e., $U_{\varepsilon}(f)=\{g \in F \mid\|g-f\|<\varepsilon\}$.

\section{Social Stability and Cyclically Stable Set}

This section defines and discusses the concept of cyclical stability. To capture the idea of social stability, we consider the following two points. First, there are no strategic considerations between any two matchings (though there may be strategic interaction within each matching situation), which reflects the assumption that individuals are anonymous and are matched randomly. Second, unlike a deviation made by a single player, a change in behavior pattern is likely to be continuous. This expresses the idea that within a small time interval, only a small portion of individuals change their strategies. In order to express these points, the notion of accessibility is given, which is similar to the one defined in Gilboa and Matsui [6]. 
DEFINITION. A strategy distribution $g$ is directly accessible from another strategy profile $f$ if there exist a continuous $p:[0,1] \rightarrow F$ which is differentiable from the right, $h:[0,1) \rightarrow F$ which is continuous from the right, and a positive number $\alpha$ such that $p(0)=f, p(1)=g$,

$$
\left(d^{+} / d t\right) p(t)=\alpha[h(t)-p(t)] \quad \text { for } \quad t \in[0,1)
$$

and

$$
h(t) \in \operatorname{Br}(p(t)) \quad \text { for } \quad t \in[0,1) .
$$

In the definition, $\left(d^{+} / d t\right) p(t)$ is the right derivative of $p$ at $t$. The definition says that the behavior pattern $p(t)$ moves in the direction of a best response to itself (toward $h(t)$ ), and it may stay at the same place only when the behavior pattern is a best response to itself. The interpretation of this definition is that only small and equal portions of individuals in each type recognize the current behavior pattern and change their strategies to those which are best responses to it. We call the function $p$ a directly accessible path from $f$ to $g$. Then a strategy distribution $g$ is said to be accessible from another distribution $f$ if at least one of the following is satisfied: (i) $g$ is directly accessible from $f$; (ii) there exists a sequence $\left\{g_{n}\right\}_{n=1}^{\infty}$ converging to $g$ such that $g_{n}$ is accessible from $f$; and (iii) $g$ is accessible from some $g^{\prime}$ which is accessible from $f$.

Using this notion of accessibility, we are now in a position to present the definition of cyclical stability.

Definition. A nonempty subset $F^{*}$ of $F^{\prime}$ is a cyclically stable set (CSS) if no $g \notin F^{*}$ is accessible from any $f \in F^{*}$, and every $g$ in $F^{*}$ is accessible from every $f$ in $F^{*}$.

A cyclically stable set is stable in the sense that once the actual behavior pattern falls into it, another strategy distribution may be realized if and only if it is within the CSS. The interpretation of this concept is as follows: for a long time, individuals seek better strategies. After they experience enough, a behavior pattern falls into a CSS, may move within it, and never leaves it. The term cyclically stable stems from the intuitive notion of cycles within a CSS. However, the paths may, of course, be much more complicated.

\section{Optimality Result and Its Proof}

This section presents the main theorem and its proof. Let $F^{* *} \subset F$ be defined by $F^{* *}=\left\{f \in F: I I(f)=\left(a_{1}, a_{2}\right)\right\}$. Note that $F^{* *}$ is closed, and that $f$ is in $F^{* *}$ if and only if $f^{k} \neq(1,1)(k \neq 0, i=1,2)$ implies that $u_{i}^{k}$ is not reached. Now, we are in a position to state the following result. 
THEOREM. $F^{* *}$ is the unique cyclically stable set in any two-by-two game of common interest with cheap-talk.

Note that the statement of the theorem does not hold in a game without cheap-talk. Consider the game of Fig. 1. If all individuals take $R$, then none has an incentive to take $L$ and get zero instead of one. Therefore, $(R, R)$ is a socially stable strategy; a fortiori, it forms a cyclically stable set as a singleton. On the other hand, in the game with cheap-talk, it is possible for individuals to cooperate and attain the optimal outcome because even if all the rest take $R$, one can take the strategy which expects a "signal" and takes $L$ if one gets the signal and remains at $R$ if one does not, which is followed by the opponent's change of the strategy to that which actually sends a signal for cooperation.

Next, we present the proof of the theorem, which consists of some lemmata. The first lemma says that for any strategy distribution, there exists another strategy distribution accessible from it which prescribes either $(L, L)$ or $(R, R)$ at each information set of the second stage.

Lemma 1. Given $f \in F$, there exists $g$ in $F$ which is accessible from $f$ such that for each $k=1,2,3,4$, either $g^{k}=(0,0)$ or $g^{k}=(1,1)$.

Proof. Given $f \in F$ and $\varepsilon>0$, we first construct $p$ and $h$ satisfying the conditions of direct accessibility such that $p^{k}(1)$ is in the $\varepsilon$-neighborhood of $(0,0)$ or $(1,1)$ for all $k=1, \ldots, 4$. As is seen from the expression of an

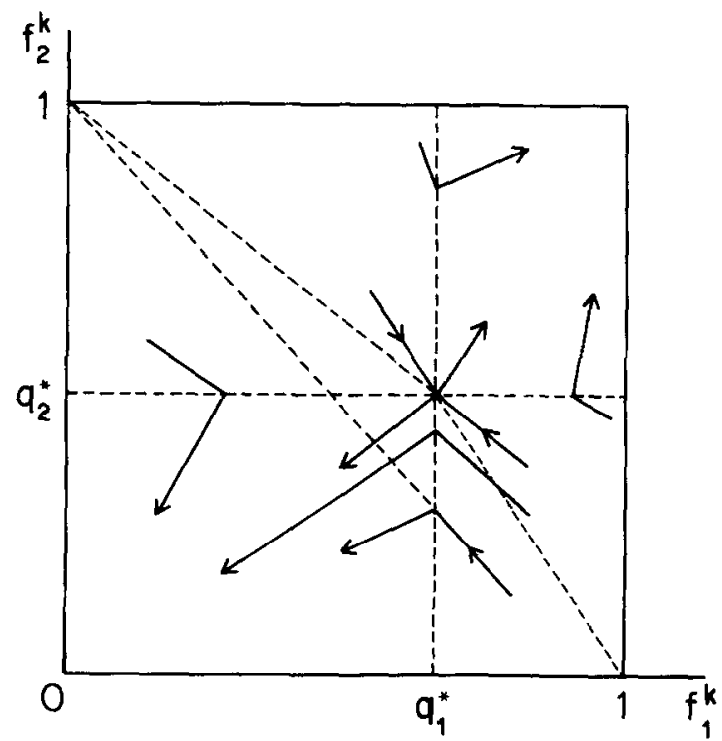

FIGURE 3 
expected payoff, $h^{k}(k \neq 0)$ can be determined independently of $p^{k^{\prime}}$ and $h^{k^{\prime}}$ for $k^{\prime} \neq k$. Let $h_{i}^{k}(k \neq 0, i=1,2)$ be defined as

$$
h_{i}^{k}(t)= \begin{cases}1 & \text { if either } p_{j}^{k}(t)>q_{j}^{*} \text { or }\left[p_{j}^{k}(t)=q_{j}^{*} \text { and } p_{i}^{k}(t)>q_{i}^{*}\right] \\ 0 & \text { otherwise. }\end{cases}
$$

Then $h_{i}^{k}(t)$ maximizes $\pi_{i}\left(h_{i}^{\prime}, p_{i}^{k}(t)\right)$ with respect to $h_{i}^{\prime}$. By letting

$$
\left(d^{+} / d t\right) p^{k}(t)=\alpha\left[h^{k}(t)-p^{k}(t)\right],
$$

$p^{k}(1)$ approaches $(1,1)$ or $(0,0)$ as $\alpha$ becomes larger. Figure 3 illustrates the dynamics for the case of $d_{i}-b_{i}>0$ for $i=1,2$.

Next, we consider the dynamics of $p^{0}(t)$. Let $H_{i}\left(p^{0}, t\right)(i=1,2)$ be defined by

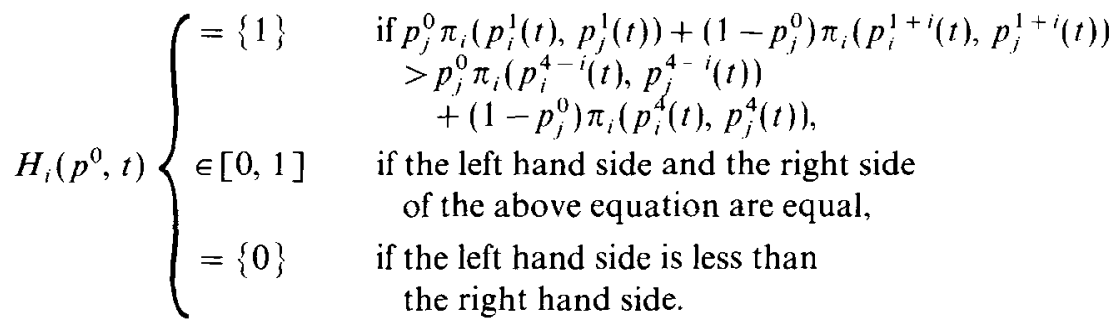

The left (resp., right) hand side of the above inequality is expected payoff of type $i$ player if he/she takes $L$ (resp., $R$ ) at $u_{i}^{0}$, i.e., chooses $f_{i}^{0}=1$ (resp., $f_{i}^{0}=0$ ). Consider the following problem of differential inclusion:

$$
\left(d^{+} / d t\right) p^{0}(t) \in \alpha\left[\left(H_{1}\left(p^{0}, t\right), H_{2}\left(p^{0}, t\right)\right)-\left(p_{1}^{0}, p_{2}^{0}\right)\right], \quad p^{0}(0)=f^{0} .
$$

Note that any $h(t)$ with $h^{0}(t) \in\left(H_{1}\left(p^{0}, t\right), H_{2}\left(p^{0}, t\right)\right)$ and $h^{k}(t)$ defined as above is in $\operatorname{Br}(p(t))$. By the existence theorem of differential inclusion (see, e.g., Aubin and Cellina [1, p. 98]), the above problem has a solution since $H_{i}\left(p^{0}, t\right)$ is upper semicontinuous and convex-valued. Therefore, for each $\varepsilon>0$, there exists a directly accessiblc path $p$ such that $p^{k}(1) \in U_{\varepsilon}((0,0)) \cup U_{\varepsilon}((1,1))$ for each $k=1,2,3,4$.

Let $\left\{\varepsilon_{n}\right\}_{n=1}^{\infty}$ and $\left\{g_{n}\right\}_{n=1}^{\infty}$ be sequences such that $\left(\varepsilon_{n}\right)$ converges to zero, and $g_{n}^{k}(1)$ is in $U_{\varepsilon_{n}}((0,0))$ or $U_{\varepsilon_{n}}((1,1))$. Then there exists a subsequence $\left(g_{n_{l}}\right)$ which is convergent. Let $g$ be the limit of $\left(g_{n_{l}}\right)$. Then $g$ is accessible from $f$ and $g^{k}=(0,0)$ or $(1,1)$ for each $k \neq 0$.

Q.E.D.

The next lemma states, by making use of Lemma 1, that for any strategy distribution, there exists another distribution in $F^{* *}$ which is accessible from it. 
Lemma 2. For any $g \in F$, there exists $f$ in $F^{* *}$ which is accessible from $g$.

Proof. From Iemma 1, it is sufficient to show that for any $g$ such that for each $k \neq 0, g^{k}=(0,0)$ or $(1,1)$, there exists $f$ in $F^{* *}$ which is accessible from $g$. Consider the case in which $g^{k}=(0,0)$ holds for all $k \neq 0$. The result is easily proven for the other cases as well. Let $g^{\prime}$ be such that $g^{\prime k}=(0,0)$ holds for $k=0$ as well as $k \neq 0$. For any $\varepsilon>0$, there exists $g^{k} \in U_{k}\left(g^{\prime}\right)$ that is directly accessible from $g$. Indeed, given $0<\varepsilon<1, p:[0,1] \rightarrow F$ defined by

$$
p^{0}(t)=\varepsilon^{t} g^{0}+\left(1-\varepsilon^{t}\right) g^{\prime 0}, \quad p^{k}(t)=(0,0)
$$

is a directly accessible path from $g$ to $\varepsilon g+(1-\varepsilon) g^{\prime}$ with $h(t)=g^{\prime}$ for all $t$ and $\alpha=-\ln \varepsilon$. Thus, $g^{\prime}$ is accessible from $g$.

Now, consider $g^{*} \in F$ with $g^{* 0}=g^{* 1}=(1,1)$ and $g^{* k}=(0,0)$ for $k=2,3,4$. Then a path $p$ defined by $p(t)=\varepsilon^{t} g^{\prime}+\left(1-\varepsilon^{t}\right) g^{*}$ is a directly accessible path from $g^{\prime}$ to $\varepsilon g^{\prime}+(1-\varepsilon) g^{*}$ with $h(t)=g^{*}$ for all $t$ and $\alpha=-\ln \varepsilon$. Indeed, $h(t) \in \operatorname{Br}(p(t))$ holds for all $t$ since we have $g^{*} \in \operatorname{Br}\left(g^{\prime}\right)$ and $g^{*} \in \operatorname{Br}\left(g^{*}\right)$ by virtue of $g^{\prime 0}=(0,0)$. Thus, a strategy distribution which attains $\left(a_{1}, a_{2}\right)$ is accessible from any strategy distribution by virtue of Lemma 1 and transitivity of accessiblity.

Q.E.D.

Now, we present the proof of the theorem.

Proof of the theorem. Since we establish the fact that for any strategy distribution $g$, there exists $f$ in $F^{* *}$ which is accessible from $g$, what we have to prove is that any two strategy distributions in $F^{* *}$ are mutually accessible and that any $g$ outside $F^{* *}$ is not accessible from any strategy distribution in $F^{* *}$. Since $F^{* *}$ is connected, and pairs of payoffs of all the elements in it are identical, it is clear that any two members in $F^{* *}$ are mutually accessible.

Next, take any $f$ in $F^{* *}$. Suppose the contrary, i.e., that there exists $g \notin F^{* *}$ which is accessible from $f$. Then there exist $p:[0,1] \rightarrow F$, $h:[0,1) \rightarrow F$, and $\alpha>0$ which satisfy the definition of direct accessiblity since $F^{* *}$ is closed. We may assume without loss of generality that $f$ is a point of departure at time 0 ; i.e., for any small $\Delta \hat{t}>0$, there exists $\Delta t<\Delta \hat{t}$ such that $p(\Delta t) \notin F^{* *}$ holds. We divide the proof into three cases.

First, suppose that $0<f_{i}^{0}<1$ holds for $i=1,2$. Then $f^{k}=(1,1)$ for $k \neq 0$. Let $0<\varepsilon<\frac{1}{2} \min \left\{f_{1}^{0}, 1-f_{1}^{0}, f_{2}^{0}, 1-f_{2}^{0}, 1-q_{1}^{*}, 1-q_{2}^{*}\right\}$. Take any $g^{\prime}$ in $U_{\varepsilon}(f)$. Since $0<g_{i}^{\prime 0}<1 \quad(i=1,2)$ and $g^{\prime k}>\left(1-q_{1}^{*}, 1-q_{2}^{*}\right)$ hold, $h^{\prime} \in \operatorname{Br}\left(g^{\prime}\right)$ implies that $h^{\prime k}=(1,1)$ for $k \neq 0$. Therefore, $\left\|p(t)-F^{* *}\right\|$ is decreasing in $t$. Thus, no path can leave $F^{* *}$ at $f$. 
Second, suppose that $0<f_{i}^{0}<1$ and $f_{i}^{0}=0$ or 1 hold. Assume without loss of generality that $0<f_{2}^{0}<1$ and $f_{1}^{0}=0$ hold. Then since $u_{i}^{1}$ and $u_{i}^{2}$ $(i=1,2)$ are not reached, $f^{1}$ and $f^{2}$ may be anything, while $f^{3}=f^{4}=(1,1)$. Again, for $g^{\prime}$ in any sufficiently small neighborhood of $f, h^{\prime} \in \operatorname{Br}\left(g^{\prime}\right)$ implies that $h_{1}^{\prime 0}=0$ whenever either $g^{\prime 1}$ or $g^{\prime 2}$ is different from $(1,1)$, which means that $\left\|p(t)-F^{* *}\right\|$ is decreasing in $t$.

Finally, suppose that $f_{1}^{0}=f_{2}^{0}=0$. Then $f^{4}=(1,1)$. Take any $g^{\prime}$ in a sufficiently small neighborhood of $f, h^{\prime} \in \operatorname{Br}\left(g^{\prime}\right)$ implies that $h_{1}^{\prime 0}=0$ if $g^{\prime 2} \neq(1,1), h_{2}^{\prime 0}=0$ if $g^{\prime 3} \neq(1,1)$, and $h^{\prime 0}=(0,0)$ if $g^{\prime 2}=g^{\prime 3}=(1,1)$ and $g^{\prime 1} \neq(1,1)$ hold. This again implies that $\left\|p(t)-F^{* *}\right\|$ is always decreasing in $t$. Other cases are proven in the same way. Thus, there is no directly accessible path from any $f$ in $F^{* *}$ to $g$ outside $F^{* *}$.

Q.E.D.

\section{Other EXAMPLes}

This section discusses some other games. First of all, the optimality result can be extended to the class of two-person (not necessarily two-bytwo) pure-coordination games with cheap-talk in which the payoffs to both players are identical. The proof is similar to the one presented in an earlier version of this paper (Matsui [9]).

Next, we consider the game of the "battle of sexes" shown in Fig. 4. It is shown that in this game, a pair of payoffs $(1.5,1.5)$ is attained in a cyclically stable set, namely that cheap-talk serves as a correlation device. Suppose that $T$ (resp., $L$ ) is taken by type 1 players (resp., type 2 players) at $u_{1}^{1}$ and $u_{1}^{4}$ (resp., $u_{2}^{1}$ and $u_{2}^{4}$ ), and $B$ (resp., $R$ ) is taken by type 1 players resp., type 2 players) at $u_{1}^{2}$ and $u_{1}^{3}$ (resp., $u_{2}^{2}$ and $u_{2}^{3}$ ); i.e., $f^{k}$ is $(1,1)$ for $k=1,4$ and $(0,0)$ for $k=2,3$. Furthermore, suppose that $f_{1}^{0}=f_{2}^{0}=\frac{1}{2}$. Similar to what we have seen in the previous section, since all the information sets are reached with positive probability, and since each player strictly prefers to keep the present action at each information set of the second stage, we conclude that $f_{i}^{k}(k \neq 0, i=1,2)$ is not changed in the neighborhood of $f^{0}$. Moreover, there is no directly accessible path for the

L

$\mathrm{R}$

\begin{tabular}{|ll|ll|}
\hline $\mathrm{L}$ & 1 & 0 & 0 \\
\hline $\mathrm{R}$ & 2 & 1 & 2 \\
\hline
\end{tabular}

FigURE 4 


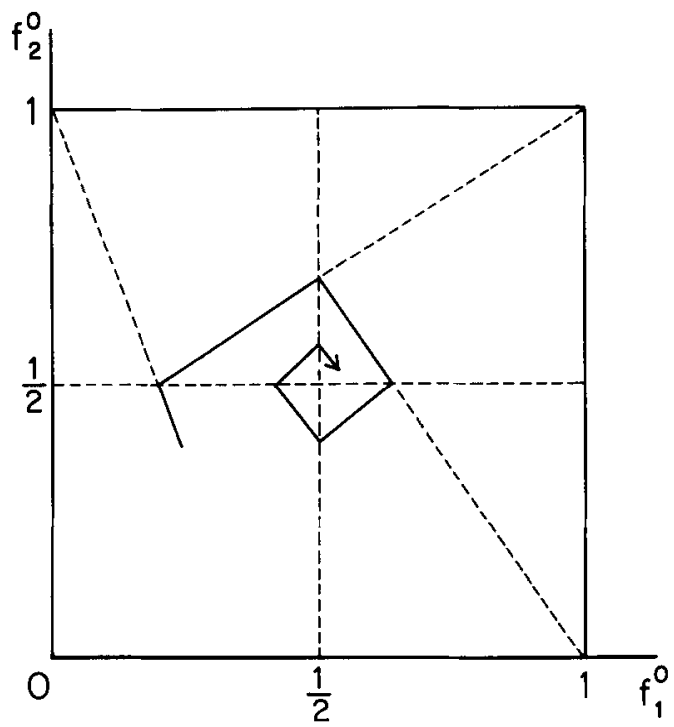

Figure 5

behavior pattern to move away from $f^{0}$ if the $f_{i}^{k \text { 's }}(k \neq 0, i=1,2)$ do not change as can be seen in Fig. 5. Thus, $\{f\}$ is a cyclically stable set as a singleton, and $\Pi(f)=(1.5,1.5)$. It has not heen proven whether this is a unique cyclically stable set or not. ${ }^{5}$

It is not always the case that Pareto efficient outcomes are selected in the course of social adjustment. See Fig. 6. One may prove that $(B, R)$ is a CSS. Indeed, in this game, $(B, L)$ is always accessible from $(T, L)$, and $(B, R)$ is also accessible from them. Therefore, a CSS consists of $(B, L)$, $(T, L),(B, R)$, and some mixtures of them. This game has a unique strongly Pareto optimal outcome. Thus, the uniqueness of the weakly, not only strongly, Pareto optimal outcome is essential for the optimality result.

L $\quad$ R

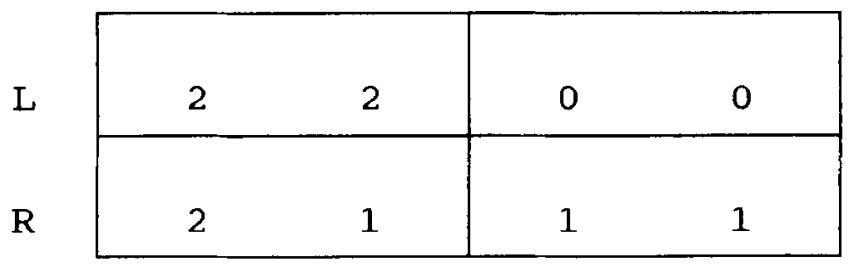

Figure 6

\footnotetext{
${ }^{5}$ One may observe that the extension of Farell's concept of neologism-proof equilibrium done in footnote 1 does not work here since either one of the players can use credible neologism to get two. It, of course, is possible that one changes the definition to cope with the problem, which, however, makes the concept less intuitive and more arbitrary.
} 


\section{Conclusion}

We have seen that cyclically stable sets predict the set of Pareto optimal outcomes to be the only solutions in two-by-two games of common interest with cheap-talk. The point of this analysis is not only that cooperation is necessarily achieved, but also that it is achieved without any presumption on the credibility of cheap-talk. A high degree of credibility of cheap-talk is attained, rather than assumed, in the societal dynamic process. In other words, "truth becoming focal" is in our analysis instead of "truth being focal" as in Farrell [5] and Rabin [13].

This result reinforces the validity of the solution concept when we consider random matching games in a large society, especially those games in which players' reasoning process alone cannot sort out a single equilibrium. Of course, as to the general applicability of the concept, we must wait for the judgment of future research.

\section{REFERENCES}

1. J. P. Aubin and A. Cellina, "Differential Inclusions," Springer-Verlag, Berlin, 1984.

2. R. Aumann And S. Sorin, Cooperation and bounded recall, Games Econ. Behat. 1 (1989), 5-39.

3. B. D. BERNHEIM, Rationalizable strategic behavior, Econometrica 52 (1984), 1007-1028.

4. B. D. Bernheim, B. Peleg, and M. D. Whinston, Coalition-proof Nash equilibria I: Concepts, J. Econ. Theory 42 (1987), 1-12.

5. J. Farrell, "Meaning and Credibility in Cheap-Talk Games," Working Paper No. 8609. University of California, Berkeley, 1986.

6. I. Gilbon AND A. Matsui, Social stability and equilibrium, Econometrica, to appear.

7. D. Kreps and R. Wilson, Sequential equilibria, Econometrica 50 (1982), 863-894.

8. A. MAtsur, Information leakage forces cooperation, Games Econ. Behav. 1 (1989), 94-115.

9. A. Matsul, "Cheap-Talk and Coorperation in the Society," Discussion Paper No. 848 . Northwestern University, 1989.

10. J. Maynard Smith and G. R. Price, The logic of animal conflict, Nature 246 (1973), 15-18.

11. J. NaSH, Non-coorperative games, Ann. Math. 54 (1951), 286-295.

12. D. G. PEARCE, Rationalizable strategic behavior and the problem of perfection, Econometrica 52 (1984), 1029-1050.

13. M. Rabin, Communication between rational agents, J. Econ. Theory 51 (1990), 144-170. 